\title{
Attitudes of physicians, nurses and pharmacists concerning the development of clinical pharmacy activities in a university hospital
}

\author{
ANDREJA ČUFAR ${ }^{1}$ \\ ALEŠ MRHAR ${ }^{2}$ \\ IGOR LOCATELLI ${ }^{*}$ \\ ${ }^{1}$ University Medical Centre Ljubljana \\ Pharmacy, SI-1000 Ljubljana, Slovenia \\ ${ }^{2}$ Faculty of Pharmacy, University of \\ Ljubljana, SI-1000 Ljubljana, Slovenia
}

Accepted September 1, 2014

\begin{abstract}
It is essential to identify the expectations of physicians and nurses regarding clinical pharmacy $(\mathrm{CP})$ services before its introduction in a hospital, because it is known that their expectations can substantially differ from the pharmacists' point of view. Agreement of leading physicians, nurses and clinical pharmacists about the importance of $\mathrm{CP}$ activities in the hospital was evaluated using five point Likert scale questionnaire. Two groups of CP activities were set; the activities related to the hospital system (first group) and the activities connected with an individual patient (second group). Total mean score of agreement of physicians with the first and second group of CP activities is 4.28 and 3.73, respectively, while these scores are lower for nurses (3.87 and 3.38 for the first and second group, respectively). Pharmacists' total mean scores are highest, 4.57 and 4.23 for the first and second group, respectively.
\end{abstract}

Keywords: clinical pharmacist, activities, physicians, nurses

In contrast to traditional pharmacy as a profession, with medicine being its point of interest, the point of interest of clinical pharmacy is the patient (1). Clinical pharmacy service is a set of activities including, but not limited to, provision and evaluation of all kinds of information regarding medicines, cooperation in planning and conducting pharmacotherapy, medication reconciliation, verification of prescribing, preparation for medicine administration, outcomes assessment, pharmacovigilance issues, and safe and cost effective medicine utilization assurance $(2,3)$.

Acceptance of a clinical pharmacist by other members of the health care team depends mainly on their individual attitude, perception and personal experience (4). The physicians' fear of losing independence and professional autonomy is one of the barriers and reasons for the negative attitude towards clinical pharmacists, perceived as inspectors of their work $(5,6)$. On the other side, there is a growing number of literature data about the positive economic impact of clinical pharmacists' activities (7).

\footnotetext{
*Correspondence; e-mail: igor.locatelli@ffa.uni-lj.si
} 
A plethora of research work has been done in order to evaluate the clinical pharmacy services after their introduction $(2,3,5,6,8-17)$. In contrast, studies exploring the stakeholders' needs, expectations, and perceptions of clinical pharmacy services before their introduction are very rare. Only a few studies investigated the perception of clinical pharmacy services by all three professional groups - pharmacists, physicians and nurses $(9,15$, 16). Dussart et al. (15) investigated satisfaction with only one particular pharmacist's service, i.e., individualized dispensing system. Gillespie et al. (11) conducted three different surveys, each designed for one profession only. Compared to the existing research, the added value of our research is that the survey was conducted before the clinical pharmacy service was introduced, it included the vast majority of all clinical pharmacy activities and the same questionnarie was used for all three professional groups.

The purpose of our research was to evaluate the positions of the leading physicians and head nurses on clinical pharmacy activities before their introduction in the hospital based on a comprehensive questionnaire containing all known clinical pharmacists' activities. Moreover, potential differences in perception and expectations from the clinical pharmacy service among pharmacists, physicians, and nurses were evaluated.

\section{EXPERIMENTAL}

\section{Clinical environment description}

The study was conducted at the University Medical Centre (UMC) Ljubljana, Slovenia. The medical centre has 2200 beds and over 100,000 hospitalizations, more than 750,000 ambulatory visits and over 250,000 functional diagnostic visits annually. There are 64 clinics, clinical departments, centres and institutes operating within the hospital. Forty-nine of them use medical preparations while performing their practice. Six out of 49 departments have negligible drug consumption, while a considerable amount of medications is used in the other 43 departments. We can therefore suppose that clinical pharmacy service would being valuable there. Hospital pharmacy supplies medications, including different galenic, magistral and parenteral preparations to clinical departments. At the time of conducting the survey there were 27 employed pharmacists, all working in the pharmacy. There was no clinical pharmacy service in hospital wards.

\section{Questionnaire (perception assessment tool) generation}

An internet search in Medline and PubMed and in the Firefox browser was performed for the keywords: clinical pharmacy, physician's attitude to pharmacist, clinical pharmacy activities, clinical pharmacy services, physician-pharmacist collaboration, physicians' acceptance of pharmacist, clinical pharmacy, health care services research, services development, consumers' attitude, consumers' perception, consumers' satisfaction, consumers' expectations. Primary and secondary sources were selected, including web pages of professional pharmaceutical organizations and other relevant data about clinical pharmacy services. These sources were used to make a list of clinical pharmacy activities, which was further used to construct a survey questionnaire. 
A. Čufar et al.: Attitudes of physicians, nurses and pharmacists concerning the development of clinical pharmacy activities in a university hospital, Acta Pharm. 64 (2014) 447-461.

\section{Questionnaire structure}

A questionnaire with the Likert measurement scale was constructed to conduct a descriptive observational study on the perception of physicians, nurses and pharmacists about the importance of each of the listed activities of clinical pharmacists. Questions/statements in the first part of the questionnaire (questions 1-17) pertain to the clinical pharmacy activities in the hospital system, while the second part of the questionnaire (questions 1836) contains activities directly connected with individual patient care. The participants had to choose the level of agreement on the Likert scale from 1 (I totally disagree) to 5 (I totally agree) with each of the listed affirmative statements in the questionnaire.

\section{Questionnaire validation}

The questionnaire was first tested on a group of pharmacists to check the scope of selected clinical pharmacy activities. In the second step, it was validated by a group of experts: a physician, a non-hospital pharmacist and an independent human resource manager.

\section{Study participants}

The questionnaire was sent by e-mail to all 27 pharmacists employed in the hospital pharmacy at the time of survey. They were asked to fill in the questionnaire only in case they wanted to practice clinical pharmacy in the future. In addition, the questionnaire was sent to 43 physicians - medical directors or heads of departments - and to their head nurses. We decided to perform this study on the basis of directed sampling, because we were interested in getting the middle management's opinion before introducting the new service. Conducting the survey among the leading hospital staff served as a kind of promotional activity for the new clinical pharmacy service. Study participants were instructed that they could freely distribute the survey to other leading physicians in the ward according to their own judgement. The participants could choose to fill in the questionnaire electronically or to print it out and fill it in manually.

The survey results were collected in an MS Excel database, together with the demographic data of each respondent: profession (physician, nurse or pharmacist), name of the clinic/clinical department, age and gender, and the level of agreement (from 1 to 5) with each statement about the clinical pharmacy activities.

\section{Statistical analysis}

Questionnaire data were presented as mean scores of agreement for each statement, for each profession separately. The differences between pharmacists, physicians, and nurses in the mean score of each item (statement agreement) were analysed using the KruskalWallis non parametric ANOVA test. For pairwise comparisons between professional groups, non parametric Mann-Whitney U test was applied with Holm's correction method for control of type I error.

Additionally, total mean score for each profession was calculated as the mean value of mean scores of all statements obtained for each participant. This was calculated separately for the first (statements regarding clinical pharmacy activities in the hospital system) and 
the second part of the questionnaire (statements regarding activities directed at the patient). In case of missing data for a statement score of a specific participant, the total mean score was still calculated on the basis of the data obtained from other statement scores of this specific participant). The differences between pharmacists, physicians and nurses in the total mean score of agreement with each statement were analysed with a one way ANOVA test. Homogeneity of variances was assessed with Levene's test. In cases of heterogeneity of variances, we assessed the differences in perception between professional groups using Welch's test. For pairwise comparisons between professional groups, post hoc tests (Scheffe's test in cases of homogeneous variances and Games-Howell's test in cases of heterogoneous variances) were used. Statistical analysis was performed in the IBM SPSS Statistics programme, version 21 (IBM Corporation, Armonk, New York, USA). Significance level was set at 0.05 .

\section{RESULTS AND DISCUSSION}

In total 40 physicians (46\% women mean age 45 years, and $54 \%$ men mean age 51 years) have filled the questionnaire. In the nursing group, all of 42 participating nurses were women, mean age was 46 years. Out of total 27 pharmacists, 13 (85\% women, mean age of women and men was 45 years and 30 years, respectively) expressed their wish to work as clinical pharmacists and filled in the questionnaire. All together, 82 responses from physicians and nurses as users of the clinical pharmacy service were analysed in comparison with 13 responses of pharmacists as potential providers of the service.

The aggregated score of agreement with all the statements about the role of a clinical pharmacist in the hospital system and his/her role in the patient-connected activities for each professional group, i.e., pharmacists, physicians and nurses, are shown in Fig. 1. There

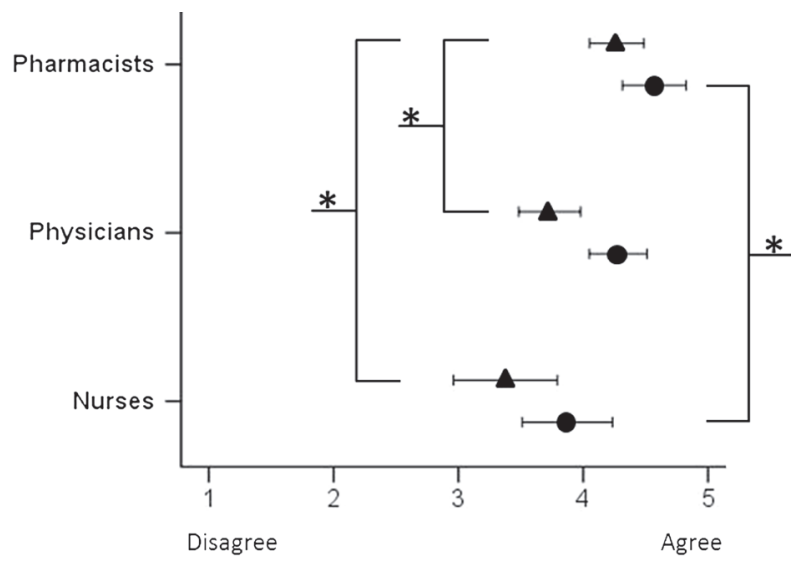

Fig. 1. Total mean score of agreement (Likert scale) with all statements about the role of a clinical pharmacist in the hospital system, for pharmacists $(n=13)$, physicians $(n=40)$ and nurses $(n=42)$. Error bars represent $95 \%$ confidence interval of the mean value. Asterisks denote statistically significant pairwise comparisons among professions, obtained separately for items appraising the roles for the hospital system (circles, 17 items) and for the patient (triangles, 19 items). 
A. Čufar et al.: Attitudes of physicians, nurses and pharmacists concerning the development of clinical pharmacy activities in a university hospital, Acta Pharm. 64 (2014) 447-461.

are significant differences in the total mean score of agreement about the role of clinical pharmacists in the hospital system between pharmacists and nurses, whereas the differences between pharmacists and physicians, and between physicians and nurses were not statistically significant. Differences in the total mean score of agreement about the role of clinical pharmacist for the patient were statistically significant when comparing pharmacists with physicians and nurses, whereas the differences between physicians and nurses were not statistically significant.

\section{The role of a clinical pharmacist in the hospital system}

The mean score of agreement with all statements about the role of clinical pharmacists in the hospital system aggregated for all three professional groups was 4.14. The nurses' total mean score was 3.87 and was the lowest, physicians ranked it 4.28 and pharmacists 4.57 (Fig. 1, circles). The differences between professional groups were statistically significant (Welch tests of equality of means: $p<0.001$ ). A statistically significant difference was found between the mean scores of pharmacists and nurses (Games-Howell test: $p<0.001$ ), but not between the mean scores of physicians and nurses, nor between pharmacists and physicians (Fig. 1). Mean scores of agreement with each statement about the role of a clinical pharmacist in the hospital system for pharmacists, physicians and nurses are presented in Table I. Among seventeen statements about the clinical pharmacists' role in the hospital system there were thirteen statements with the mean score $>4$ for all participants. Eleven statements in this group reached the mean score $>4$ for pharmacists and physicians.

Pharmacists ranked their agreement higher (mean score ranged from 4.23 to 5 ) than physicians (mean score ranged from 3.69 to 4.80) for fourteen statements and higher than nurses (mean score from 3.63 to 4.55 ) for fifteen statements. For two statements, a statistically significant difference was found only between pharmacists and nurses.

Statistically significant differences between the mean scores of physicians and nurses were found only for the statement, regarding provision of information about medicines during different kinds of internal educational courses. All of the 13 pharmacists totally agreed with five statements, namely statements number 1, 3, 4, 6 and 16 (Table I).

Pharmacists' participation in creating pharmacotherapeutic guidelines, its designing guidelines and recommendations for improvement of drug administration procedures, and providing all kinds of drug information by the pharmacist were statements with the highest mean scores in the survey. These are the most valuable activities of clinical pharmacists reported also by other authors $(2,3)$.

Hospital pharmacists are usually passive in providing all kinds of information about medicines to other health care professionals, i.e., they do that only when asked. In contrast, clinical pharmacists do that more systematically and in a proactive way during organized meetings in the wards. Despite the statistically significant difference between pharmacists and physicians concerning these statements, there is considerably high scores in the physicians' group too, which indicates their high expectations for a systematic approach to providing information about medicines by clinical pharmacist. Also Mysak (16) and Chevalier and Neville (3) found that ear-marking of an assigned clinical pharmacist for a particular ward and a systematic approach in providing clinical pharmacy activities resulted in higher level of satisfaction physicians' and nurses'. For that reason, the high mean score for 


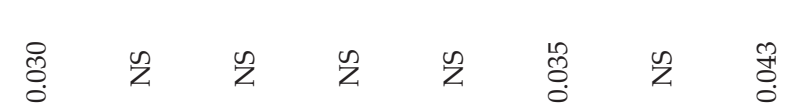

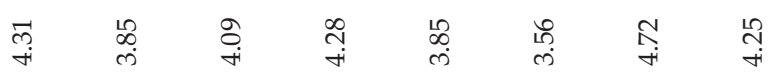

㚐

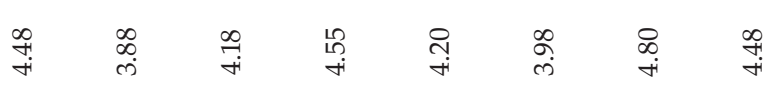

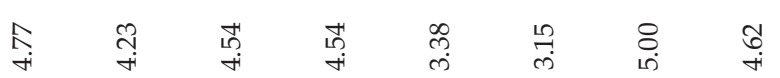

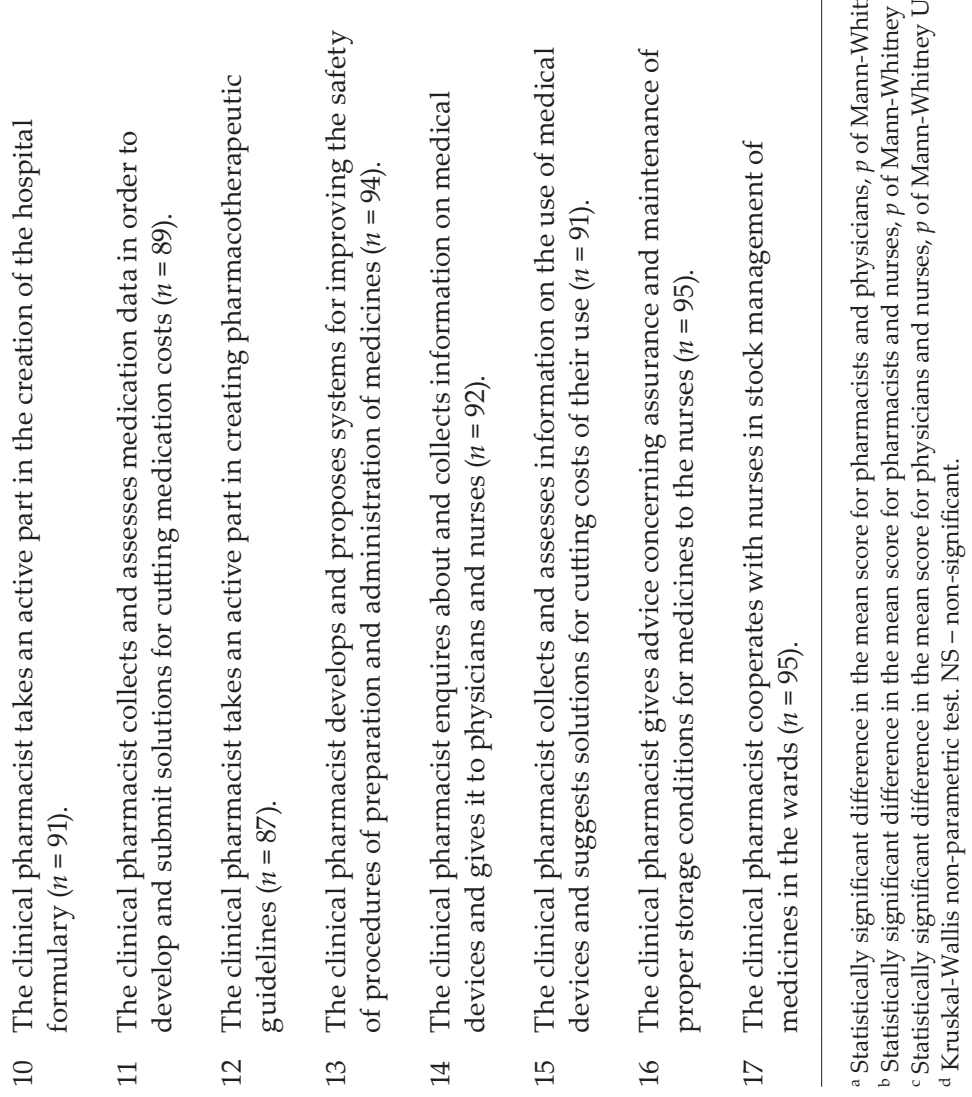


A. Čufar et al.: Attitudes of physicians, nurses and pharmacists concerning the development of clinical pharmacy activities in a university hospital, Acta Pharm. 64 (2014) 447-461.

these statements in the physicians' group is expected. When comparing the scores for similar statements concerning the pharmacists provision of information to the nurses (statements number 2, 5 and 7), the mean scores were much lower for all three professional groups. It is interesting that physicians ranked the statements about clinical pharmacists' providing information about medicines to the nurses (statements number 2, 5 and 7) the lowest. On the other side, in the nurses' group, the mean scores for some statements concerning providing information about accessibility and interchangeability of drugs to the nurses (statements number 2 and 5) are higher for nurses than for physicians. Based on this, we can conclude that it is important for a clinical pharmacist to provide information about medicines to physicians and to nurses, providing both professional groups believe clinical pharmacists should provide information to their profession with the provision of clinical pharmacy services.

For two statements (14 and 15), the mean score of agreement of pharmacists was lower than the mean score of agreement of physicians and nurses. Although the differences for statement number 14 are not statistically significant, it is important to note that they both relate to the pharmacist's role in dealing with medical devices. In the UMC Ljubljana, medical devices are provided directly by the purchasing department rather than by the hospital pharmacy. This is the reason why pharmacists did not recognize their role in dealing with medical devices and they ranked respective statements low. Nurses also ranked these two statements considerably low, 3.64 and 3.26, respectively, but physicians' mean scores were 4.20 and 3.98, which shows higher expectations of physicians concerning this topic. As far as we know, in other hospitals, medical devices are provided in the same way as medicines, i.e., by the hospital pharmacy. There were two statements that directly addressed clinical pharmacists' role in cost containment (statements number 11 and 15). It is interesting that for these two statements the mean scores of agreement were among the lowest for all three professional groups. Despite many reports about a positive economic impact of clinical pharmacists' activities in the literature (18-27), physicians and nurses found those clinical pharmacists' activities less important. Budgeting and cost containment in the hospital is the responsibility of managers rather than pharmacists. Taking into account the previously mentioned findings, the managing personnel need quality information provided by clinical pharmacists so that they can make appropriate decisions concerning costs. Clinical pharmacists exert their role concerning cost containment through their clinical activities including providing of quality information. This is concordant with the conclusion of Touchette et al. (7) that clinical pharmacy services are generally considered cost effective and provide a good benefit-cost ratio.

\section{The role of the clinical pharmacist with respect to the patient}

The total mean score of agreement with the statements about the role of a clinical pharmacist with respect to the patient was 3.65 for all three professional groups. Pharmacists ranked their mean score 4.23 , physicians scored the role of the pharmacist with respect to the patient 3.73 and nurses 3.38 (Fig. 1). The differences between professional groups were statistically significant (Welch tests of equality of means: $p<0.001$ ). A statistically significant difference was found between the mean scores of pharmacists and physicians (GamesHowell test: $p=0.0041$ ) and between the mean scores of pharmacists and nurses (GamesHowell test: $p<0.001$ ), but not between the mean scores of physicians and nurses (Fig. 1). 
A. Čufar et al.: Attitudes of physicians, nurses and pharmacists concerning the development of clinical pharmacy activities in a university hospital, Acta Pharm. 64 (2014) 447-461.

Table II gives the mean scores of agreement with the statements about the role of clinical pharmacists with respect to the patient for each professional group, namely, pharmacists, physicians, and nurses. Among nineteen statements about the role of the clinical pharmacist with respect to the patient, eleven statements showed a statistically significant differences of the mean score of agreement between the three professional groups (Table II). For four statements, the mean scores of agreement of pharmacists were also statistically significantly different from the mean scores of agreement of physicians and nurses. For three statements, the difference was statistically significant only between the mean scores of agreement of pharmacists and nurses, but not between the mean scores of agreement of pharmacists and physicians. There were also five statements with statistically significant difference between the mean scores of agreement of physicians and nurses.

Among the statements about the role of the clinical pharmacist with respect to the patient the statement number 36: »The clinical pharmacist can (has the power to) interchange the prescribed medicine with its pharmaceutical alternative without proven bioequivalence, but has to inform the physician about the interchange as soon as possible", has obtained the lowest mean score of agreement of all, for all professional groups, although this activity of the pharmacist has achieved very positive outcomes somewhere else $(2,27)$. Actually, therapeutic interchange is a frequent activity of US-based clinical pharmacists and its frequency is increasing in some other countries, as well.

Physicians showed clear disagreement (mean score 2.00) also with the statement number 35: »The clinical pharmacist can (has the power to) interchange the prescribed medicine with its pharmaceutical equivalent without proven bioequivalence, but has to inform the physician about the interchange as soon as possible« and with the similar statement about interchanging of essentially similar medicines (statement number 34, mean score 2.50). Also, pharmacists were not in favour of performing the activities described in statements 35 and 36 (mean scores 3.15 and 2.62). Actually, all three statements concerning the power of the pharmacist to interchange the prescribed drugs with other suitable items without prior confirmation of the physician (statements 34, 35 and 36) have gained very low mean scores of agreement of physicians (mean scores 2.5, 2.00 and 1.93, respectively) and nurses (mean scores 2.55, 2.40 and 2.23). In contrast, the mean score of agreement of pharmacists with the statement number 34: » The clinical pharmacist can (has the power to) interchange essentially similar medications without consultation with the physician, when this is necessary for cost- or accessibility-related reasons concerning the prescribed medicine « was considerably high (4.23), whereas the mean scores of agreement of pharmacists with the statements about independently interchanging medications without evidence of being bioequivalent (statements 35 and 36) were lower for interchanging of pharmaceutical equivalents (3.15) and for interchanging of pharmaceutical alternatives (2.61). Research of Kjeldsen et al. (2) showed very similar results. One possible explanation for disagreement of physicians with all three statements about independent interchanging of medicines by clinical pharmacists is that they overlooked the differences between the kinds of drugs to be interchanged. Actually, our hospital pharmacy usually supplies only one of the essentially similar medicines and the interchange of the ordered medication is performed in the pharmacy. In the ward, prescribed drugs are interchanged with other bioequivalent drugs by the nurses on the basis of information from the pharmacy. For the planning of future activities of clinical pharmacists in the hospital, it is important to be aware of the physicians' strong negative attitude towards pharmacists' competence for independent inter- 


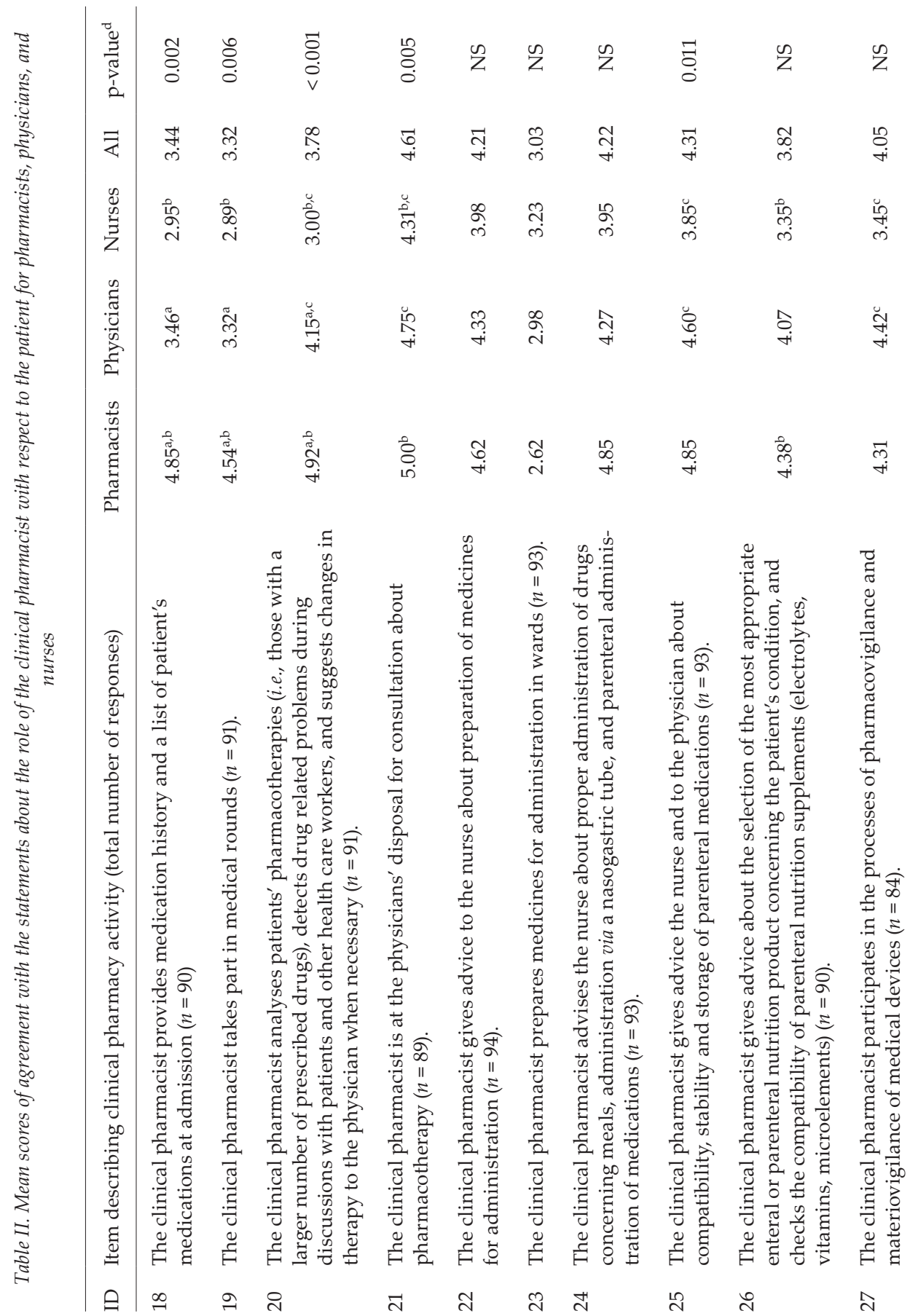




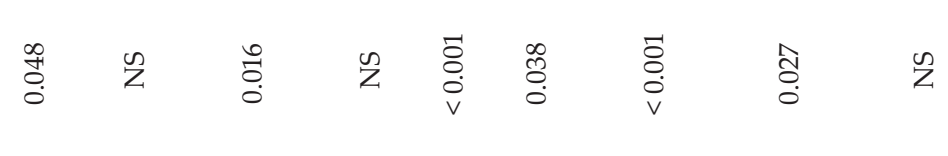

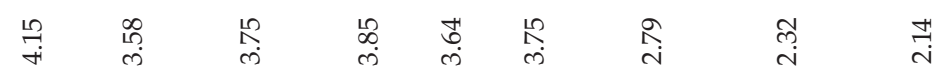

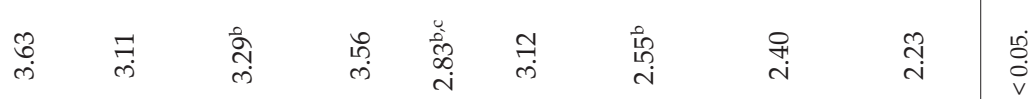

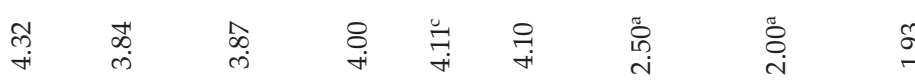

马)

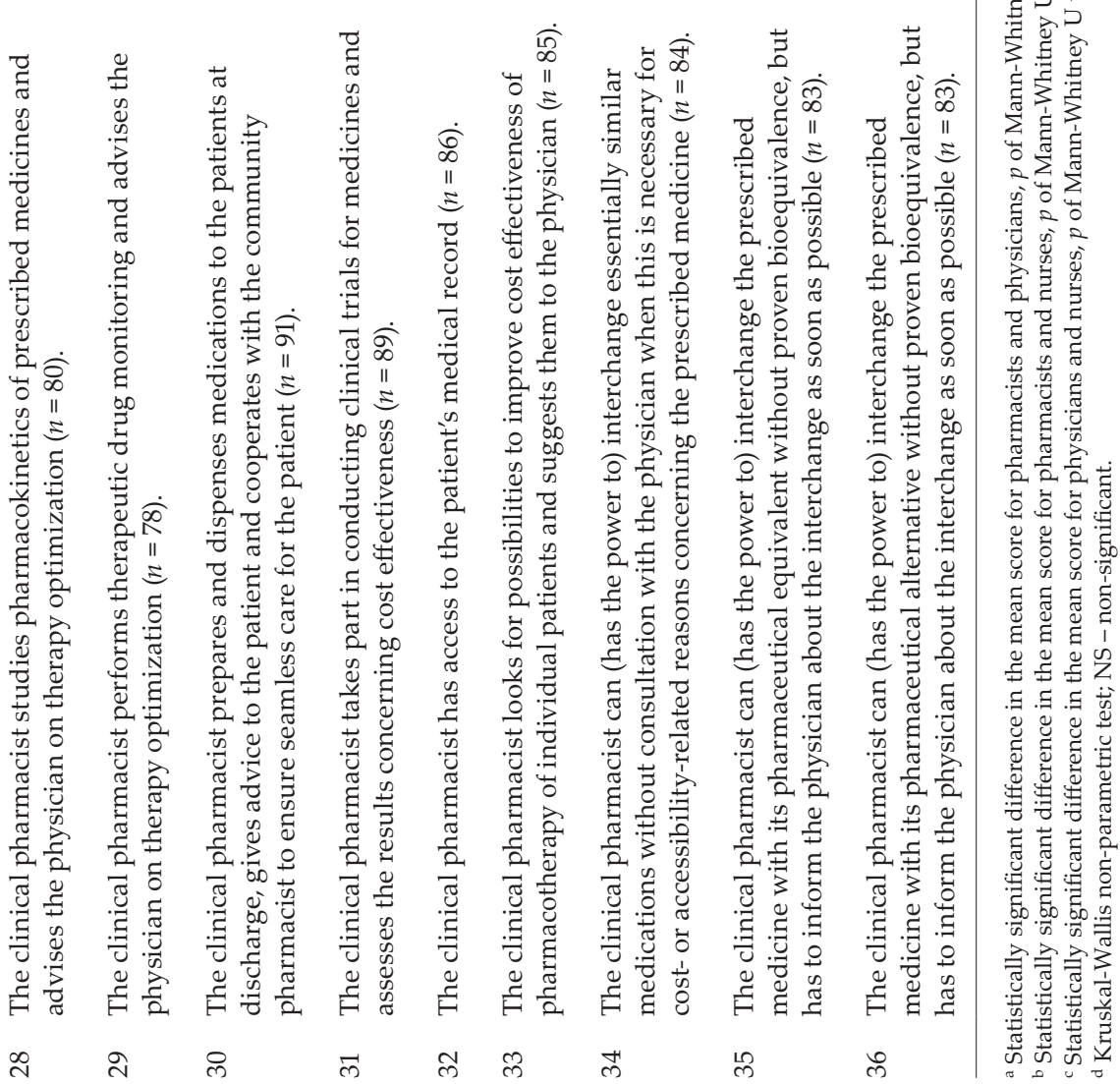


A. Čufar et al.: Attitudes of physicians, nurses and pharmacists concerning the development of clinical pharmacy activities in a university hospital, Acta Pharm. 64 (2014) 447-461.

change of prescribed medicines. According to the literature, there are some other activities of clinical pharmacists that have demonstrated a very positive impact on the outcomes of medication therapy, among them participation of the pharmacist in medical rounds, taking medication history, medication reconciliation and giving advice to the patient at discharge, etc. $(2,3,27)$. Notwithstanding, our survey has established significantly lower mean scores of agreement of physicians and nurses with the respective statements. Physicians expressed a surprisingly low support (mean score 3.32) to participation of clinical pharmacists in medical rounds, while nurses were even against it (mean score 2.89). On the other hand, physicians expressed a high level of agreement with some other statements concerning clinical pharmacists' participation in activities aimed at achieving good and safe pharmacotherapy, as seen for statements number 20 (mean score 4.15), 21 (mean score 4.75), 27 (mean score 4.42), 28 (mean score 4.32) and 33 (mean score 4.10). They also support clinical pharmacists' access to patients' medical records, described in statement 32 (mean score 4.11). It is interesting that nurses expressed their negative opinion concerning this statement (mean score 2.83). Also, the agreement of nurses with some other statements about clinical pharmacists' cooperation with physicians is considerably lower compared to physicians. These are statements number 20 (mean score 3.00), 25 (mean score 3.85), 27 (mean score 3.45), 32 (mean score 2.83) and 33 (mean score 3.12). Nurses showed a rather reserved attitude even to typically pharmaceutical activities like preparation (statements 22 and 23) of medications, compatibility issues (statements 24, 25 and 26), dispensing of medications at discharge (statement 30 ) and pharmacovigilance (statement 27), when perform in the wards. This may be due to the nurse fear that clinical pharmacists would be a kind of inspection over their professional field. Among the statements about the role of clinical pharmacists with respect to the patient, nurses ranked $>4$ only one statement (number 21 , mean score 4.31).

\section{Limitations of the research}

Of more than 1100 physicians and more than 3500 nurses in the UMC Ljubljana, only 40 head physicians and 42 head nurses were included in the survey. Sampling was deliberately directed because we wanted to research the perception of and attitude towards the clinical pharmacy service of the middle management health care professionals who lead and direct activities in the wards. In spite of the small sample, we expected to get better information about the possibilities of introducting the clinical pharmacy service into the hospital in this way. The survey was anonymous and we can assume its validity was not diminished by possible social desirability bias.

In addition, the number of participating pharmacists is considerably low. Nevertheless, all thirteen pharmacists, who were interested in performing clinical pharmacy services, answered the questionnaire, so we determined a $100 \%$ response rate for pharmacists. Moreover, for statements 27, 28, 29, 34, 35, and 36, more than $10 \%$, of the data was missing (number of responses was 84 or lower), which occurred mainly in the nurses' group. The terms in these statements are specifically connected with the pharmacy profession and may not be adequately understood by other health care professionals, especially nurses. This is the possible reason for the considerably high rate of missing data. Additionally, all three statements about the power of the pharmacist to independently interchange the prescribed medication (statements 34-36) were, despite considerable differences in professional content (bioequivalent vs. non-bioequivalent medications), ranked very similarly by physi- 
cians and nurses. The possible reason could be the mind-set bias because of small differences in the text of statements and the position bias because of the sequential order of these three statements. We suggest to other authors who would like to use our questionnaire to merge these three statements.

\section{CONCLUSIONS}

In this research, we identified the most important clinical pharmacy activities from the users', i.e., physicians' and nurses' point of view, and from the pharmacists' on the other side. There is a substantial difference in the opinion about the importance of a particular activity between pharmacists as providers and physicians and nurses as users. In the majority of cases, pharmacists ranked their agreement with the statements higher (4.57 in the hospital system group and 4.23 in the patient set of statements) than physicians (4.28 and 3.73 , respectively) and nurses (3.78 and 3.38, respectively). Nurses expect clinical pharmacists to provide information about medicines to the nursing staff, including advice concerning storage of medication. Physicians' expectations are much more clinically oriented. They expect clinical pharmacists to analyse and consult patients' pharmacotherapies, detect drug related problems and suggest changes or optimization of the therapy. However, physicians' perception of the role of the clinical pharmacist is more traditional and they are not in favour of passing the right to perform independent interventions of pharmacotherapy to clinical pharmacists. Nevertheless, they all agree that pharmacists should have access to medical records.

Performing research and analysis of the future »market stakeholders' « attitude towards the newly planned service is a more managerial and proactive approach, which can avert providers from performing unnecessary or even unwanted activities. We conducted the survey using the same set of questions for all three professional groups. This allowed us to compare the answers concerning a particular clinical pharmacy activity among different professions. This can be a useful starting point for other researchers in this field.

\section{REFERENCES}

1. R. R. Miller, History of clinical pharmacy and clinical pharmacology, J. Clin. Pharmacol. 21 (1981) 195-197; DOI: 10.1002/j.1552-4604.1981.tb05699.x.

2. L. J. Kjeldsen, T. B. Jensen and J. J. Jensen, Physicians' evaluation of clinical pharmacy revealed increased focus on quality improvement and cost savings, Dan. Med. Bul. 58 (2011) 1-5; DOI: A4261.

3. B. Chevalier and H. L. Neville, Evaluating clinical pharmacy services on a surgical patient-care area: a nurses' satisfaction survey, Int. J. Pharm. Pract. 19 (2011) 61-69; DOI: 10.1111/j.2042-7174.2010.00076.x.

4. Y. Punekar, S. W. Lin and J. Thomas, 3rd, Progress of pharmacist collaborative practice: status of state laws and regulations and perceived impact of collaborative practice, J. Am. Pharm. Assoc. 43 (2003) 503-510; DOI: 10.1331/154434503322226257.

5. F. M. Alkhateeb, K. A. Clauson, R. McCafferty and D. A. Latif, Physician attitudes toward pharmacist provision of medication therapy management services, Pharm World Sci: PWS 31 (2009) 487-493; DOI: 10.1007/s11096-009-9304-1. 
A. Čufar et al.: Attitudes of physicians, nurses and pharmacists concerning the development of clinical pharmacy activities in a university hospital, Acta Pharm. 64 (2014) 447-461.

6. A. J. Zillich, R. P. McDonough, B. L. Carter and W. R. Doucette, Influential characteristics of physician/pharmacist collaborative relationships, Ann. Pharmacother. 38 (2004) 764-770; DOI: 10.1345/ aph.1D419.

7. D. R. Touchette, F. Doloresco, K. J. Suda, A. Perez, S. Turner, Y. Jalundhwala, M. C. Tangonan and J. M. Hoffman, Economic evaluations of clinical pharmacy services: 2006-2010, Pharmacotherapy 34 (2014) 771-793; DOI: 10.1002/phar.1414.

8. S. Giberson, S. Yoder and M. P. Lee, Improving patient and health system outcomes through advanced pharmacy practice, A Report to the U.S. Surgeon General, Office of the Chief Pharmacist. U.S. Public Health Service, Washington 2011, pp. 15-45.

9. J. A. Sulick and D. S. Pathak, The perceived influence of clinical pharmacy services on physician prescribing behavior: a matched-pair comparison of pharmacists and physicians, Pharmacotherapy 16 (1996) 1133-1141; DOI: 10.1002/j.1875-9114.1996.tb03042.x.

10. T. M. Rauch, The perceptions of army physicians and nurses on the relative importance of clinical pharmacy services, Mil. Med. 147 (1982) 391-395; DOI: 10.1111/j.1365-2753.2008.00990.x.

11. U. Gillespie, C. Morlin, M. Hammarlund-Udenaes and M. Hedstrom, Perceived value of wardbased pharmacists from the perspective of physicians and nurses, Int. J. Clin. Pharm. 34 (2012) 127-135; DOI: 10.1007/s11096-011-9603-1.

12. G. R. Bailie and B. Romeo, New York State primary care physicians' attitudes to community pharmacists' clinical services, Arch. Intern. Med. 156 (1996) 1437-1441; DOI: 10.1001/archinte.156.13.1437.

13. A. V. Law, M. D. Ray, K. K. Knapp and J. K. Balesh, Unmet needs in the medication use process: perceptions of physicians, pharmacists, and patients, J. Am. Pharm. Assoc. 43 (2003) 394-402; DOI: 10.1331/154434503321831111.

14. L. M. Tahaineh, M. Wazaify, A. Albsoul-Younes, Y. Khader and M. Zaidan, Perceptions, experiences, and expectations of physicians in hospital settings in Jordan regarding the role of the pharmacist, Res. Soc. Adm. Pharm. 5 (2009) DOI: 63-70; 10.1016/j.sapharm.2008.05.003.

15. C. Dussart, S. Dussart, D. Almeras, I. Camal and G. Grelaud, Assessing user satisfaction and hospital pharmacy practice: application to an individualized dispensing system in a French military teaching hospital, J. Eval. Clin. Pract. 15 (2009) 252-256; DOI: 10.1111/j.1365-2753.2008.00990.x.

16. T. M. Mysak, C. Rodrigue and J. Xu, Care providers' satisfaction with restructured clinical pharmacy services in a tertiary care teaching hospital, Can. J. Hosp. Pharm. 63 (2010) 105-112; DOI: 10.4212/ cjhp.v63i2.893.

17. F. J. Ritchey, M. R. Raney and T. D. Keith, Physicians' opinions of expanded clinical pharmacy services, Am. J. Pub. Health 73 (1983) 96-101; DOI: 10.2105/AJPH.73.1.96.

18. S. T. McMullin, J. A. Hennenfent, D. J. Ritchie, W. Y. Huey, T. P. Lonergan, R. A. Schaiff, M. E. Tonn and T. C. Bailey, A prospective, randomized trial to assess the cost impact of pharmacist-initiated interventions, Arch. Intern. Med. 159 (1999) 2306-2309; DOI: 10.1001/archinte.159.19.2306.

19. T. W. Nesbit, K. M. Shermock, M. B. Bobek, D. L. Capozzi, P. A. Flores, M. C. Leonard, J. K. Long, M. A. Militello, D. A. White, L. D. Barone, M. P. Goldman and D. A. Kvancz, Implementation and pharmacoeconomic analysis of a clinical staff pharmacist practice model, Am. J. Health Syst. Pharm. 58 (2001) 784-790.

20. C. A. Bond and C. L. Raehl, Clinical and economic outcomes of pharmacist-managed antimicrobial prophylaxis in surgical patients, Am. J. Health Syst. Pharm. 64 (2007) 1935-1942; DOI: 10.2146/ ajhp060631.

21. R. E. Ariano, R. H. Demianczuk, R. G. Danzinger, A. Richard, H. Milan and B. Jamieson, Economic impact and clinical benefits of pharmacist involvement on surgical wards, Can. J. Hosp. Pharm. 48 (1995) 284-289.

22. G. T. Schumock, P. D. Meek, P. A. Ploetz and L. C. Vermeulen, Economic evaluations of clinical pharmacy services-1988-1995, Pharmacotherapy 16 (1996) 1188-1208; DOI: 10.1002/j.1875-9114.1996. tb03049.x 
23. M. Kilcup, D. Schultz, J. Carlson and B. Wilson, Postdischarge pharmacist medication reconciliation: impact on readmission rates and financial savings, J. Am. Pharm. Assoc. 53 (2013) 78-84; DOI: 10.1331/JAPhA.2013.11250.

24. H. L. Neville, B. Chevalier, C. Daley, L. Nodwell, C. Harding, A. Hiltz, T. MacDonald, C. Skedgel, N. J. MacKinnon and K. Slayter, Clinical benefits and economic impact of post-surgical care provided by pharmacists in a Canadian hospital, Int. J. Pharm. Pract. 22 (2014) 216-222; DOI: 10.1111/ ijpp.12058.

25. T. E. Richardson, C. L. O'Reilly and T. F. Chen, A comprehensive review of the impact of clinical pharmacy services on patient outcomes in mental health, Int. J. Clin. Pharm. 36 (2014) 222-232; DOI: 10.1007/s11096-013-9900-y.

26. J. C. Samp, D. R. Touchette, J. S. Marinac and G. M. Kuo, Economic evaluation of the impact of medication errors reported by U.S. clinical pharmacists, Pharmacotherapy 34 (2014) 350-357; DOI: 10.1002/phar.1370.

27. P. J. Kaboli, A. B. Hoth, B. J. McClimon and J. L. Schnipper, Clinical pharmacists and inpatient medical care: a systematic review, Arch. Intern. Med. 166 (2006) 955-964; DOI: 10.1001/ archinte.166.9.955. 